\title{
Simulation of Glazing Behavior in Fires using Computational Fluids Dynamics and Spectral Radiation Modeling
}

\author{
SIAKA DEMBELE ${ }^{1}$, RICARDO ROSARIO ${ }^{1}$, JENNIFER WEN $^{1}$, PAUL WARREN $^{2}$, and STUART \\ DALE $^{2}$ \\ ${ }^{1}$ Faculty of Engineering, Kingston University, Friars Avenue, London SW15 3DW, UK. \\ ${ }^{2}$ Pilkington European Technical Centre, Lathom Ormskirk, Lancashire 140 5FU, UK.
}

\begin{abstract}
The performance and behavior of glazing systems have significant impact on fire growth and development. In the typical scenario of a glass pane exposed to fire, radiation is the predominant mode of heat transfer. In previous studies, the present authors have developed and validated a spectral radiation heat transfer model based on the Discrete Ordinates Method (SDOM) which accounts for the glass spectral properties (e.g. emissivity, transmissivity) and the diffuse nature of radiation incident on the glazing. In order to model the dynamic interaction between a glass pane and fire, the SDOM has been implemented in the CFD code FDS 5.0 and the new code (FDS-SDOM) is evaluated. The first part of FDS-SDOM validation study reports a comparative analysis between FDS-SDOM, the original (unmodified) version of FDS (OFDS) and the exact solutions for varying absorption coefficients typical of glass. The comparative study, in terms of radiative heat flux, shows that FDS-SDOM provides results closer to the exact solutions in comparison to OFDS when the absorption coefficient is varied from 0.1 to $100 \mathrm{~m}^{-1}$ (maximum error less than $1 \%$ for FDSSDOM against $13 \%$ for FDS). This provides further justification for the need to account for varying spectral properties of material such as glass and the diffuse nature of radiation in calculations. In the second part of the validation study, FDS-SDOM is applied to two experimental fire and glass scenarios with the aim of predicting the transient temperature distribution in the glass. Relatively good agreements are found between the code's predictions and the experimental data. The work demonstrates the good potential of combining the CFD approach with advanced spectral radiation modeling for fire and glazing studies.
\end{abstract}

KEYWORDS: CFD, FDS, glazing, glass, fire, spectral radiation, discrete ordinates model.

\section{NOMENCLATURE LISTING}
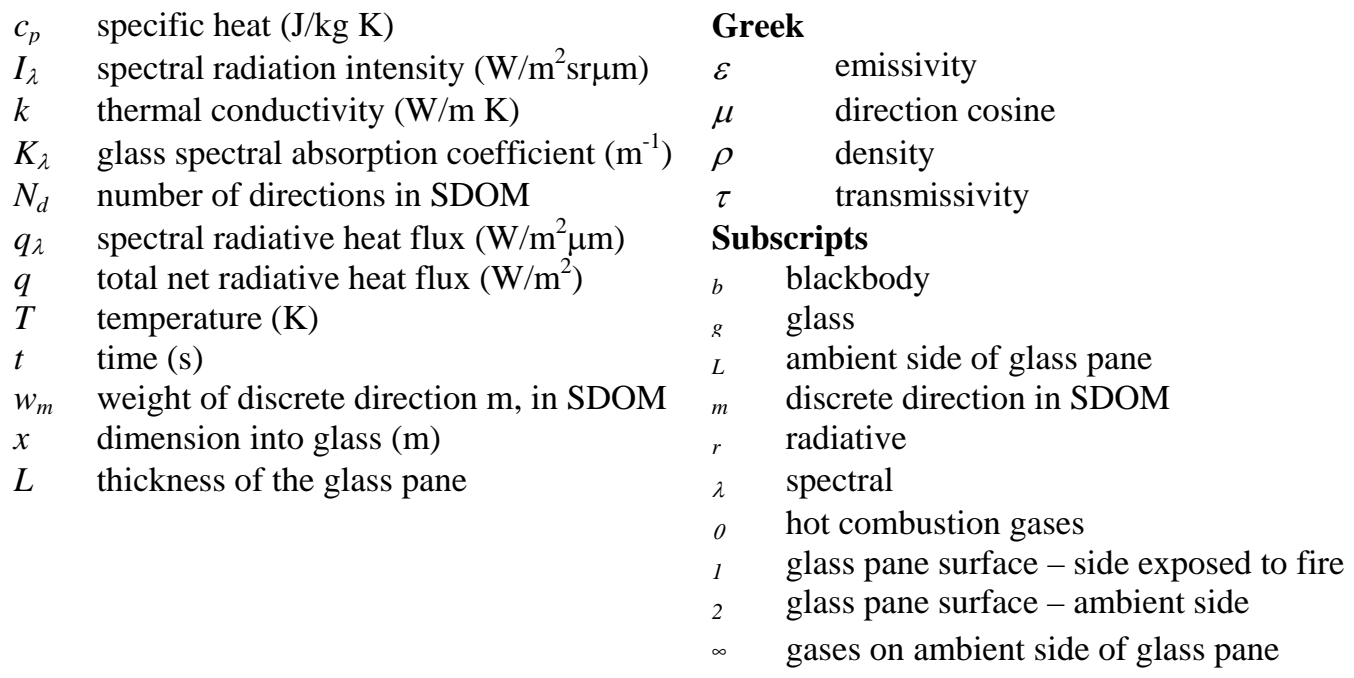

\section{INTRODUCTION}

Ventilation is one of the major factors that influence fire development. The breakage and fallout of a window or door glass pane affects ventilation and the severity of fires. The early works of Emmons [1] and Pagni [2] have provided an understanding of the glass breaking problems and established a breaking criterion based on the glass temperature increase in the middle of the pane [2]. Many theoretical and experimental studies have been carried out on the performance of window glass in fires (e.g. [3, 4]). Radiation is the predominant mode of heat transfer in fire and glazing studies. Pagni and Joshi [5] have 
developed the code BREAK 1 which models heat transfer by treating the glass as a distributed mass that absorbs radiation through its thickness with non-linear radiative boundary conditions. Although the majority of heat transfer models employed in fire/glass studies have their own merits such as the simplicity of implementation, they do not account for the spectral properties of glass (e.g. emissivity, transmissity) and the diffuse nature of incident radiation. To address these shortcomings, Dembele et al. [6, 7] have developed a spectral radiation model based on the discrete ordinates method. The validation studies of the radiation model show that the approach provides good prediction of glass temperatures and time to breakage [6]. Prescribed gas temperatures extracted from experimental data were used in simulations reported in [6] as the flow field was not computed. In the previous studies by the authors, the dynamic interaction between fluid flow, combustion (fire) and the glass pane was not accounted for as it was important to assess the radiation module independently before any coupling with fluid dynamics. With the aim of developing a tool coupling the dynamic interaction between the fire and glazing, the spectral radiation model developed by the authors has been implemented into Version 5.0 of the CFD code FDS (Fire Dynamics Simulator) developed at the National Institute of Standards and Technology (NIST), USA. The work presented in this article mainly reports on the preliminary validation studies of the Kingston University's version of the modified FDS code referred to as FDS-SDOM.

\section{MATHEMATICAL MODEL AND NUMERICAL DETAILS}

The transient temperature distribution is important in predicting the behavior of a glass pane subjected to heat from flames. The temperature is derived from the energy equation:

$$
\rho \cdot c_{p} \frac{\partial T}{\partial t}(x, t)=k \frac{\partial^{2} T}{\partial x^{2}}(x, t)-\nabla \cdot q_{r}
$$

The radiative source term, $\nabla \cdot q_{r}$, is determined from the solution of the radiative transfer equation using the spectral discrete ordinates method.

\section{Spectral Discrete Ordinates Radiation Method (SDOM)}

The spectral radiative transfer equation (RTE) in one-dimensional geometry for glass is:

$$
\mu \frac{\partial I_{\lambda}(x, \mu)}{\partial x}=-K_{\lambda}(x) \cdot I_{\lambda}(x, \mu)+K_{\lambda}(x) I_{b \lambda}[T(x)]
$$

The Discrete Ordinates Method (DOM) approach is based on the separation of the angular dependence from the spatial dependence of the radiation intensity in the RTE. For a glass pane exposed to fire radiation, with the SDOM approach, Eq. 2 becomes for a discrete direction m:

$$
\mu_{m} \frac{\partial I_{\lambda m}(x)}{\partial x}=K_{\lambda}(x) \cdot\left[I_{b \lambda}[T(x)]-I_{\lambda m}(x)\right]
$$

The boundary conditions to solve Eq. 3 for a glass pane exposed to fire in a compartment for example are:

$$
I_{\lambda m}=\varepsilon_{\lambda, g} I_{b \lambda}\left(T_{1}\right)+2 \cdot\left(1-\varepsilon_{\lambda, g}-\tau_{\lambda, g}\right) \sum_{m^{\prime}=1, \mu^{\prime}<0}^{N_{d}} \mu_{m^{\prime}} w_{m^{\prime}} I_{\lambda m^{\prime}}(0)+\varepsilon_{\lambda, 0} \cdot I_{b, \lambda, 0}\left(T_{0}\right) \quad \text { at } \quad \mathrm{x}=0
$$

and

$$
I_{\lambda m}=\varepsilon_{\lambda, g} I_{b \lambda}\left(T_{2}\right)+2 \cdot\left(1-\varepsilon_{\lambda, g}-\tau_{\lambda, g}\right) \sum_{m^{\prime}=1, \mu^{\prime}>0}^{N_{d}} \mu_{m^{\prime}} w_{m^{\prime}} I_{\lambda m^{\prime}}(L)+\varepsilon_{\lambda, \infty} \cdot I_{b, \lambda, \infty}\left(T_{\infty}\right) \quad \text { at } \mathrm{x}=\mathrm{L}
$$

The number of directions, $\mathrm{N}_{\mathrm{d}}$, for DOM applications in 1D geometry is typically 20 . The SDOM takes into account the spectral properties of the glass since actual emissivity and transmissivity of glass strongly vary 
with the wavelength [8]. The approach also handles the diffuse nature of incident fire radiation on the glass pane.

The spectral and total radiative heat fluxes in the glass are respectively given by $q_{\lambda}(x)=\sum_{m=1}^{N_{d}} w_{m} \mu_{m} I_{\lambda m}(x)$

and $q(x)=\sum_{\text {all }} q_{\Delta \lambda}(x) \Delta \lambda$.

The total radiative source term, $\nabla \cdot q_{r}$ in Eq. 1 , is obtained by differentiation of the total heat flux.

\section{CFD code FDS 5.0 and SDOM Implementation}

As underlined above, to predict the dynamic interaction between the glazing and fire, a CFD tool is required. The CFD code used to implement SDOM is the version 5.0 of FDS, the Fire Dynamics Simulator developed at the National Institute of Standards and Technology (NIST) in the USA [9]. FDS solves the Navier-Stokes equations for low Mach number ( $\mathrm{Ma}<0.3)$. The numerical algorithm employed is an explicit predictor/corrector scheme, second order accurate in space and time, using a direct Poisson solver. Turbulence is treated by means of Large Eddy Simulation (LES), via the Smagorinski sub-grid scale model. For combustion, a mixture fraction model is used. The code has been widely used by the international fire community and undergone various validations for fire applications. The Centre for Fire and Explosion Studies at Kingston University was one of the first university groups to adopt the code for some of its fire research work and has since devoted considerable effort to carry out further development and validations to the code in various applications [10].

Thermal radiation in the flow field is computed in FDS 5.0 by the finite volume technique on the same grid as the flow solver. However in the original version of FDS (OFDS), radiative transfer calculation in solids such as glass is based on a gray "two-flux" model which assumes the radiative intensity constant inside the "forward" and "backward" hemispheres [9]. Although this approximation may be reasonable for some solid materials, for glass it is more problematic for at least two reasons: the spectral properties are not accounted for, and the "two-flux" model has its own limitations mainly due to the assumption of constant intensity in each hemisphere [11]. Work has therefore been undertaken by the authors to implement the 1D SDOM into FDS 5.0 as an alternative to the gray "two-flux" model for glazing studies. The modified code is referred to as FDS-SDOM. For this implementation, the gray diffusive wall boundary condition in the OFDS was replaced with a spectral boundary condition relevant to glass in FDS-SDOM as:

$I_{w, \lambda}(s)=\varepsilon_{\lambda} I_{b w, \lambda}+\frac{1-\varepsilon_{\lambda}-\tau_{\lambda}}{\pi} \underset{s^{\prime} \cdot n_{w}<0}{\int I_{w, \lambda}}\left(s^{\prime}\right)\left|s^{\prime} \cdot n_{w}\right| d \Omega+\tau_{\lambda} I^{*}{ }_{w, \lambda}$

\section{RESULTS AND DISCUSSION}

\section{Some Validation Results of the SDOM Approach}

The SDOM has been validated independently of the flow field in previous studies by the authors using experimental data from the literature [6, 7]. Among other scenarios, the experiment of Skelly et al. [12] which consisted of a rectangular compartment ( $1 \mathrm{~m} \times 1.2 \mathrm{~m} \times 1.5 \mathrm{~m}$ high) was considered. The glass window assembly measured $0.5 \mathrm{~m} \times 0.28 \mathrm{~m}$. The tested glass was typical window glass of $2.4 \mathrm{~mm}$ thick with an unexposed edge measurement of $0.025 \mathrm{~m}$ around the entire vent opening perimeter [12]. Burning hexane liquid provided the fire in the compartment. Two different size trays were considered: $20 \mathrm{~cm} \times 20$ $\mathrm{cm}$ and $30 \mathrm{~cm} \times 20 \mathrm{~cm}$. Figure 1 presents for the $20 \mathrm{~cm} \times 20 \mathrm{~cm}$ pool fire, the experimental data and the simulation results obtained with the SDOM approach [6]. The temperature profiles of the hot upper layer are measured close to the central point of the glass window, and used as prescribed input data for the boundary condition in SDOM ( $\mathrm{T}_{0}$ in Eq. 4 ) - the flow field is not computed. The temperatures denoted by "Tests 4, 5, 6" represent the temperature profiles of the exposed side (fire side) of the glass pane measured in three repeated tests [12]. The calculated temperature profiles of the exposed side of the glass pane are in good agreement with the experimental data. The relative difference or error, between the SDOM predictions and the averaged experimental data do not exceed $3 \%$. 


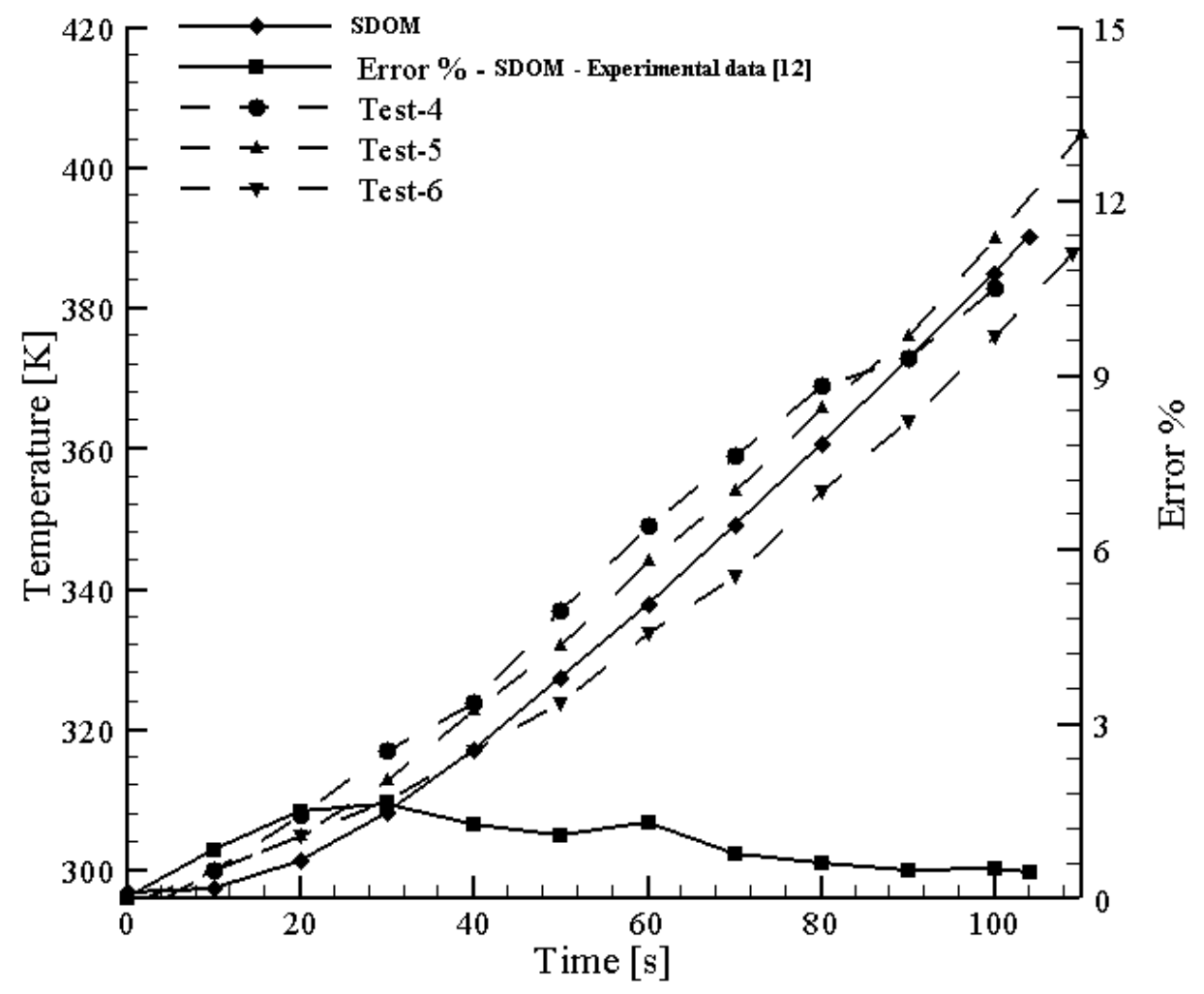

Fig. 1. Glass surface temperature predicted by SDOM and experimental data from [12].

\section{Validation Study 1 - Comparative Study between FDS-SDOM, OFDS and Exact Solutions}

The first part of the validation study of FDS-SDOM is based on a scenario for which exact heat flux solutions could be calculated. The scenario consists of a wall with an internal temperature of $1273.15 \mathrm{~K}$ and the ambient temperature is $10 \mathrm{~K}$. The wall thickness is $0.1 \mathrm{~m}$ and the absorption coefficient is varied to cover a range from 0.1 to $100 \mathrm{~m}^{-1}$ (i.e. 0.001 to $1 \mathrm{~cm}^{-1}$ ). The range covers the bulk of the glass absorption spectrum as shown in Fig. 2 [13], although the wall is not exactly glass. The emissivity, conductivity and specific heat are equal to one and the material density $1000 \mathrm{~kg} / \mathrm{m}^{3}$. For this scenario, the exact solutions for radiative flux in the wall are the analytical solutions of plane layer emission [14]:

$q=I_{b}\left[1-2 E_{-} 3(\tau)\right]$ where $I_{b}=\sigma T^{4} \sigma \cdot T^{4}$ and $E_{-} 3(\tau)^{E_{-} 3(\tau)}$ is the third-order exponential integral function of optical depth $\tau$

For comparison, the heat flux in the wall was also calculated using the original version of FDS (OFDS) based on gray "two-flux" approach for radiation calculations in solids. 


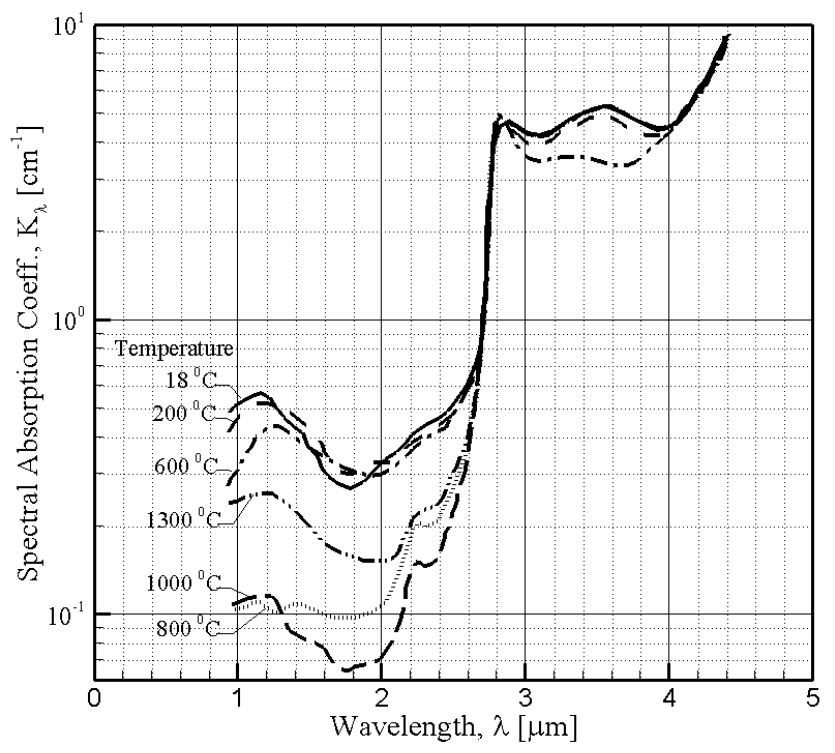

Fig. 2. Spectral absorption coefficient of window glass [13].

Table 1 presents the predicted solutions obtained with FDS-SDOM, OFDS and the exact solutions. FDSSDOM calculations are based on 100 nodes in the wall and 20 directions in SDOM. FDS-SDOM predictions are consistently close to the exact solutions and are stable. The relative differences between the two sets of data (FDS-SDOM and exact solutions) do not exceed $1 \%$. In contrast OFDS predictions show large fluctuations compared to the exact solutions with a $13 \%$ maximum relative difference. The better predictions obtained with FDS-SDOM can be explained by the ability of the SDOM approach to account for more solid angles in space to treat diffuse radiation contrary to the "two-flux" approximation based on only two hemispheres with constant radiation intensities in each. In terms of computing times, FDS-SDOM requires $10 \%$ more time than OFDS for the scenarios investigated. This validation scenario demonstrates the capability of FDS-SDOM to predict heat flux distributions for different absorptions coefficients relevant to glass, with relatively good accuracy and little computational overhead.

Table 1. Heat fluxes computed by FDS-SDOM, OFDS and exact solutions.

\begin{tabular}{|c|c|c|c|c|c|}
\cline { 2 - 6 } \multicolumn{1}{c|}{} & \multicolumn{5}{c|}{ Absorption Coefficient $\left[\mathrm{m}^{-1}\right]$} \\
\cline { 2 - 6 } & 0.10 & 1.00 & 5.00 & 10.00 & 100.00 \\
\hline $\begin{array}{c}\text { Exact Heat Flux } \\
{\left[\mathrm{kW} / \mathrm{m}^{2}\right]}\end{array}$ & 2.897000 & 24.940300 & 82.945700 & 116.2891 & 148.9698 \\
\hline $\begin{array}{c}\text { Heat Flux Predicted } \\
\left.\text { FDS-SDOM [kW/m }{ }^{2}\right]\end{array}$ & 2.902142 & 25.106795 & 82.628688 & 115.72419 & 148.15574 \\
\hline $\begin{array}{c}\text { Relative error } \\
\text { FDS-SDOM \& Exact } \\
\text { solution[\%] }\end{array}$ & 0.2 & 0.7 & 0.4 & 0.5 & 0.5 \\
\hline $\begin{array}{c}\text { Heat Flux Predicted } \\
\left.\text { OFDS [kW/m }{ }^{2}\right]\end{array}$ & 2.949532 & 26.979710 & 93.897304 & 128.41184 & 148.97023 \\
\hline $\begin{array}{c}\text { Relative error } \\
\text { OFDS \& Exact solution[\%] }\end{array}$ & 1.8 & 8.2 & 13.2 & 10.4 & 0.0003 \\
\hline
\end{tabular}




\section{Validation Study 2 - Application of FDS-SDOM to Experimental Glazing-Fire Scenarios}

The second validation study is based on the experimental scenarios of Harada et al. [15] and Skelly et al. [12]. The experiments in [15] were carried out to predict the time to first crack occurrence and/or breaking time of a glass pane. Different heat fluxes in the range 2.7 to $9.7 \mathrm{~kW} / \mathrm{m}^{2}$ were imposed to the glass by changing the distance between the glazing pane and a propane-fired radiant panel. In the independent validation of SDOM (uncoupled with fluid flow), for the heat flux of $5.48 \mathrm{~kW} / \mathrm{m}^{2}$, the experimental and predicted times to first crack occurrence were the same (207 s) [6]. The measured and SDOM predicted times to first crack were respectively $144 \mathrm{~s}$ and $147 \mathrm{~s} \mathrm{(2 \%} \mathrm{difference)} \mathrm{for} 6.69 \mathrm{~kW} / \mathrm{m}^{2}$, and $90 \mathrm{~s}$ and $94 \mathrm{~s} \mathrm{(4}$ $\%$ difference) for $9.11 \mathrm{~kW} / \mathrm{m}^{2}$ [6].

FDS-SDOM is employed to simulate the scenario of Harada et al. [15]. In calculations, the spectral range [0.1 - $200 \mu \mathrm{m}$ ] is considered and the spectral emissivity, absorption coefficient and transmissivity of glass are used. Figure 3 shows the transient temperature distribution on the exposed glass surface for an incident heat flux of $6.69 \mathrm{~kW} / \mathrm{m}^{2}$ obtained with FDS-SDOM. Transient temperatures profiles were not presented in [15], SDOM transient predictions [6] are used instead as "reference" data. The predictions of FDS-SDOM are in relatively good agreement with the reference temperatures when the spectral radiation model is coupled with FDS.

It is of interest to investigate how OFDS based on gray "two-flux" model would predict temperature distribution on the glass surface. As calculations in solids by OFDS are gray, gray calculations are carried out by varying the absorption coefficient assumed gray in the range $0.01-1000 \mathrm{~m}^{-1}$ (i.e. 0.0001 to $10 \mathrm{~cm}^{-1}$ ) to encompass the whole glass absorption coefficient spectrum (Fig. 2). OFDS predictions are shown in Fig. 3 with different absorption coefficients $\left(\mathrm{k}=0.1\right.$ to $\left.1000 \mathrm{~m}^{-1}\right)$. Transient temperature predicted by OFDS show strong fluctuations with the different absorption coefficients and do not agree with the reference data. This implies that the application of OFDS is clearly problematic for real fire/glazing scenarios where spectral glass properties should be included in calculations for accuracy. For the same experimental scenario in [15], Fig. 4 presents the glass surface temperatures obtained with FDS-SDOM as well as the reference data for three different fire heat fluxes: $5.48,6.69$ and $9.11 \mathrm{~kW} / \mathrm{m}^{2}$. Overall the two sets of data are in a relatively good agreement for the three heat fluxes considered.

The second scenario considered for the validation analysis of FDS-SDOM is based on the compartment fire experiment of Skelly et al. [12]. In contrast to the independent radiation model application of SDOM (Fig. 1), the fluid dynamics calculations are performed by FDS and dynamically coupled with SDOM for radiative source terms and temperature calculations in the glazing. Figure 5 depicts the geometry adopted for this scenario. For the computations, a total of $30(\mathrm{X})$ × $30(\mathrm{Y})$ × $30(\mathrm{Z})$ meshes were employed in $90 \%$ of the compartment volume. For the remainder of the volume, a more refined mesh based on 30x30x90 grids is used close to the glass window for more accurate predictions of the gas temperature distribution near the wall and glass window. The mesh resolution inside the glass window is 20 points across the glass thickness. A preliminary grid sensitivity analysis has shown that simulations solutions are grid independent for the mesh structure adopted for this particular scenario. The burning fuel is hexane and two different pool sizes are considered in the simulations: $20 \mathrm{~cm}$ x $20 \mathrm{~cm}$ and $30 \mathrm{~cm}$ x $20 \mathrm{~cm}$.

Figures $6 \& 7$ show the transient temperature distribution of the exposed glass surface, predicted by FDSSDOM, respectively for the $20 \mathrm{~cm} \times 20 \mathrm{~cm}$ and $30 \mathrm{~cm} \times 20 \mathrm{~cm}$ pool fires. The repeated experimental data denoted by "Tests 4, 5, 6, 1, 2, 3" [12] are also presented for comparison. FDS-SDOM predictions are closer to temperatures measured in "Test 6" $(20 \mathrm{~cm}$ x $20 \mathrm{~cm}$ pool) and "Test 1 " (30 cm x $20 \mathrm{~cm}$ pool) although there is a slight temperature under prediction in the first $20 \mathrm{~s}$. As the measurement errors were not provided in [12] it is difficult to discuss further these under-predictions and more scenarios should be investigated. FDS-SDOM calculations are overall in good agreement with the measured temperatures. 


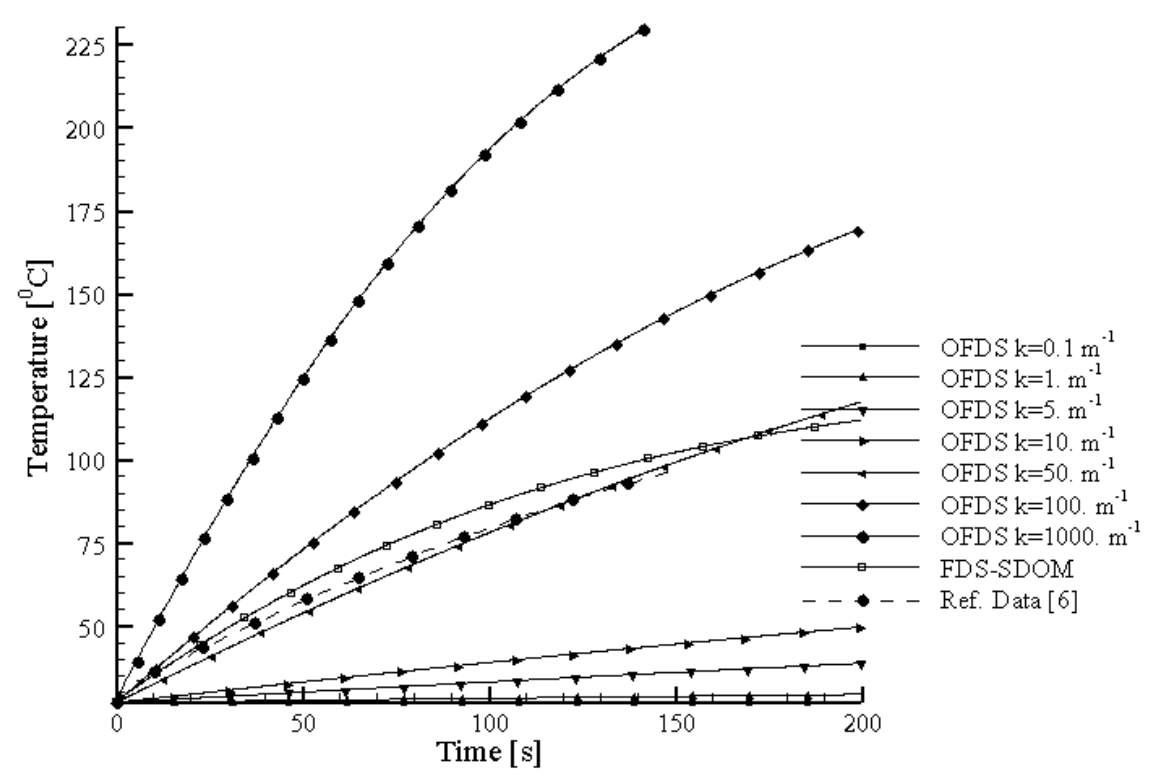

Fig. 3. Glass surface temperature predicted with FDS-SDOM, OFDS for the experimental scenario in [15] Heat flux of $6.69 \mathrm{~kW} / \mathrm{m}^{2}$

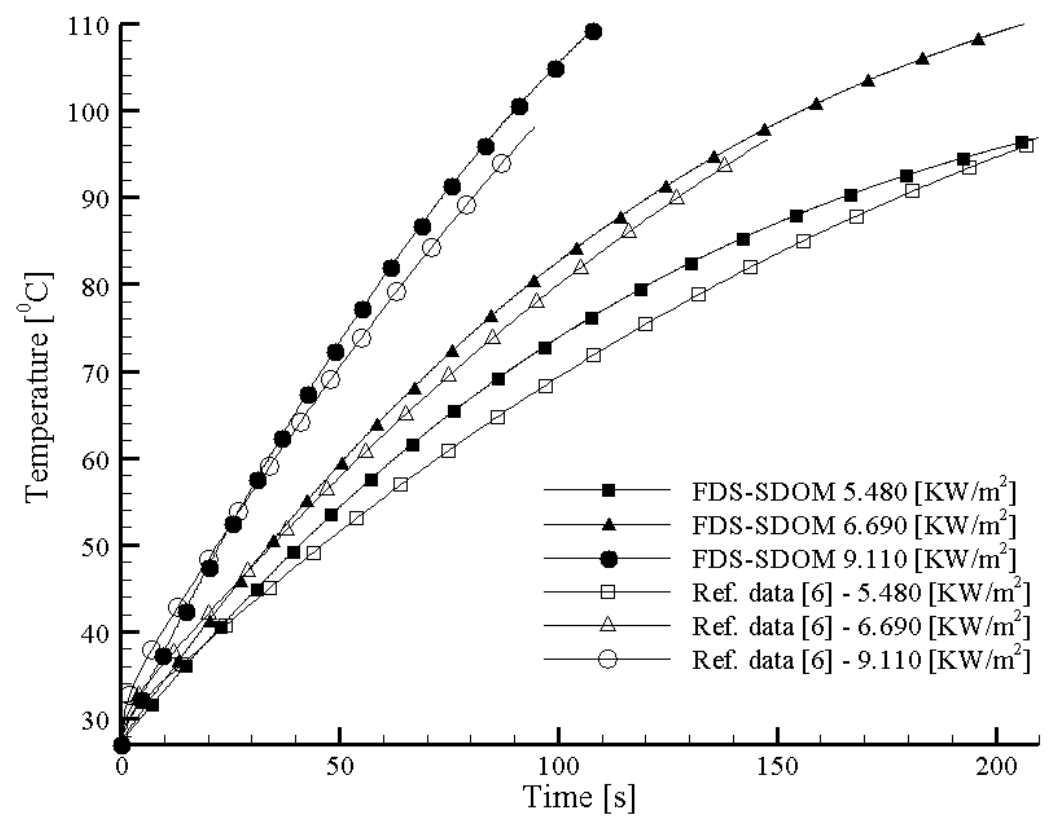

Fig. 4. Glass surface temperature predicted with FDS-SDOM for the experimental scenario in [15] for different heat fluxes. 


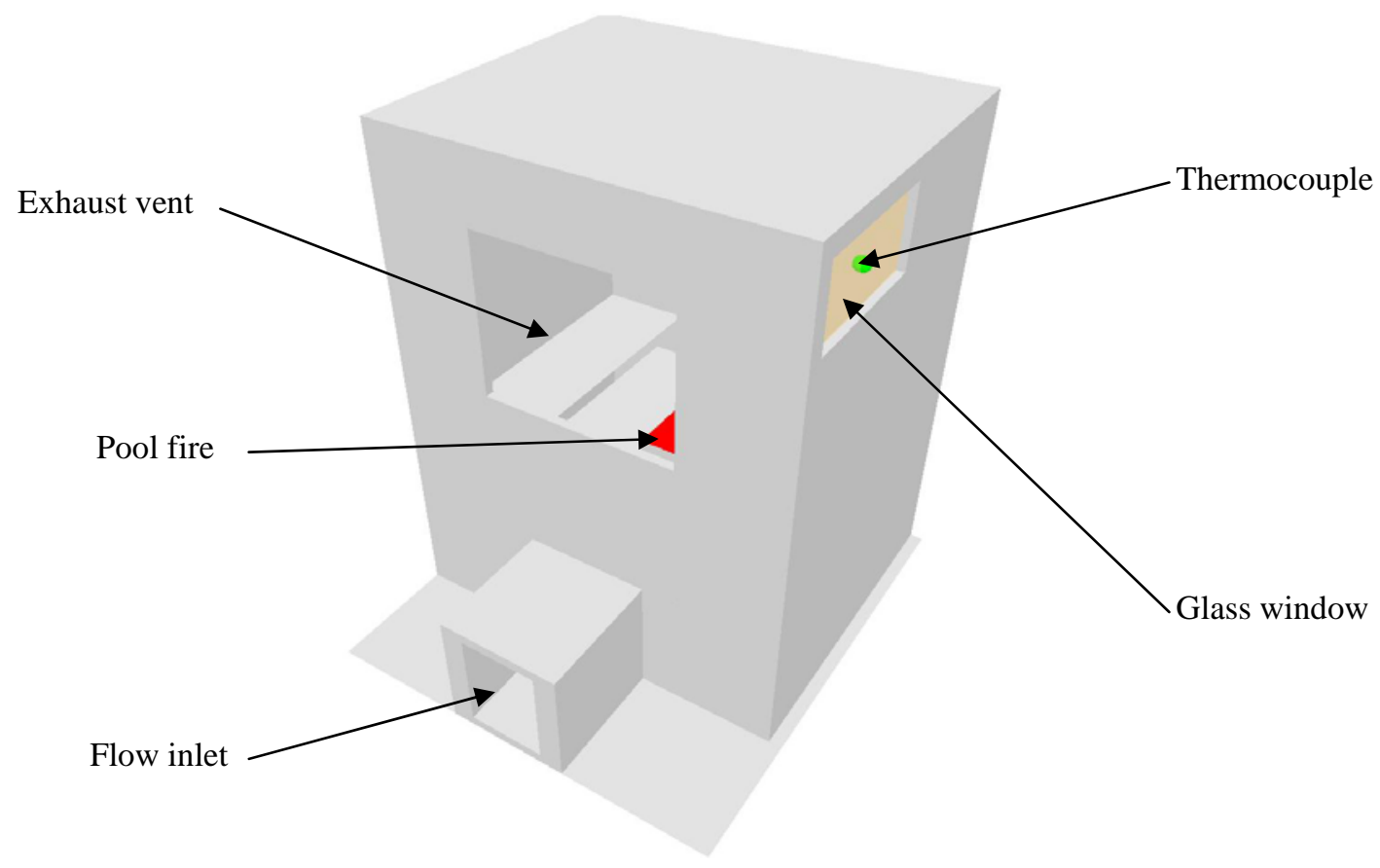

Fig. 5. Geometry for FDS-SDOM application to the compartment fire scenario in [12].

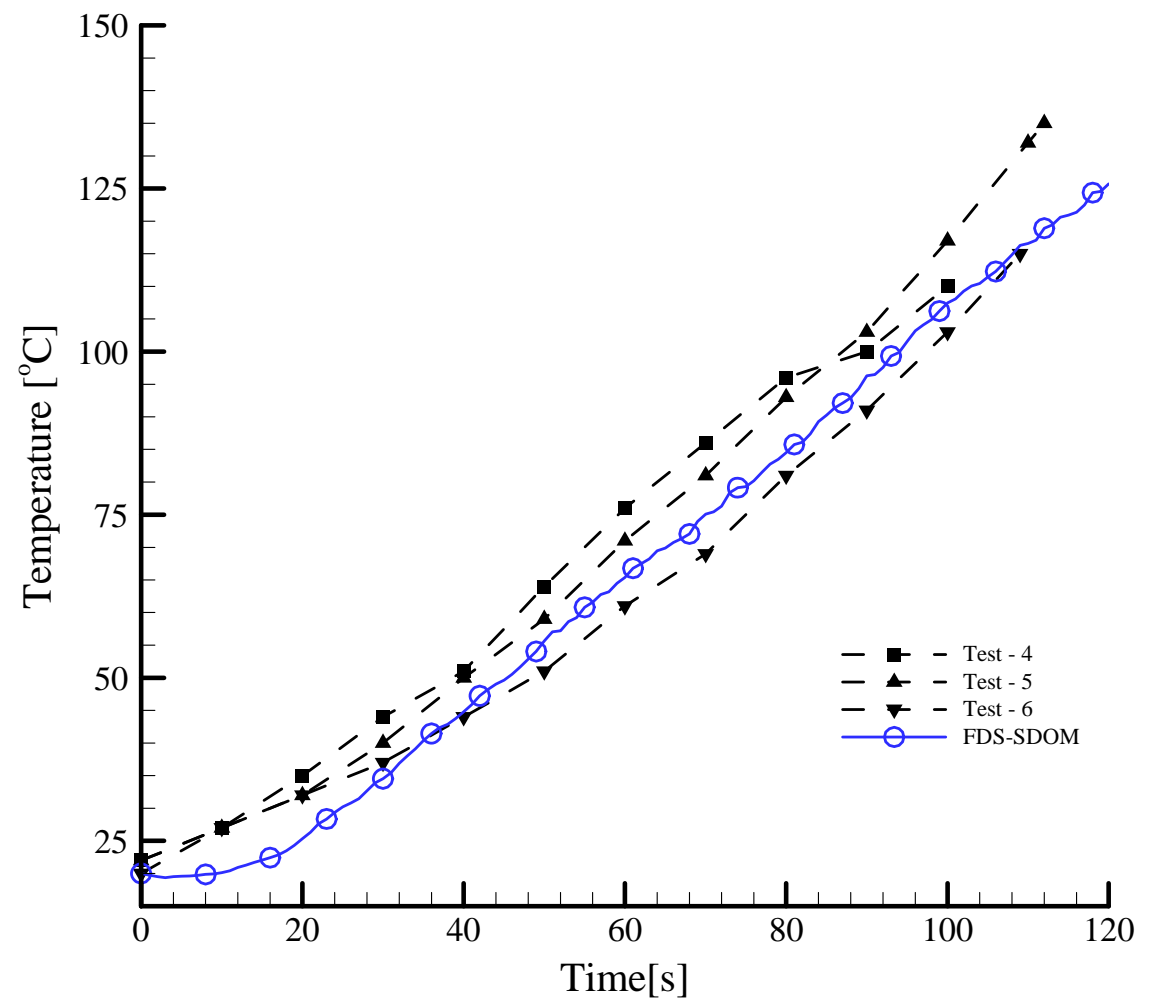

Fig. 6. Glass surface temperature predicted by FDS-SDOM and experimental data from [12]. Pool fire 20x20 cm. 


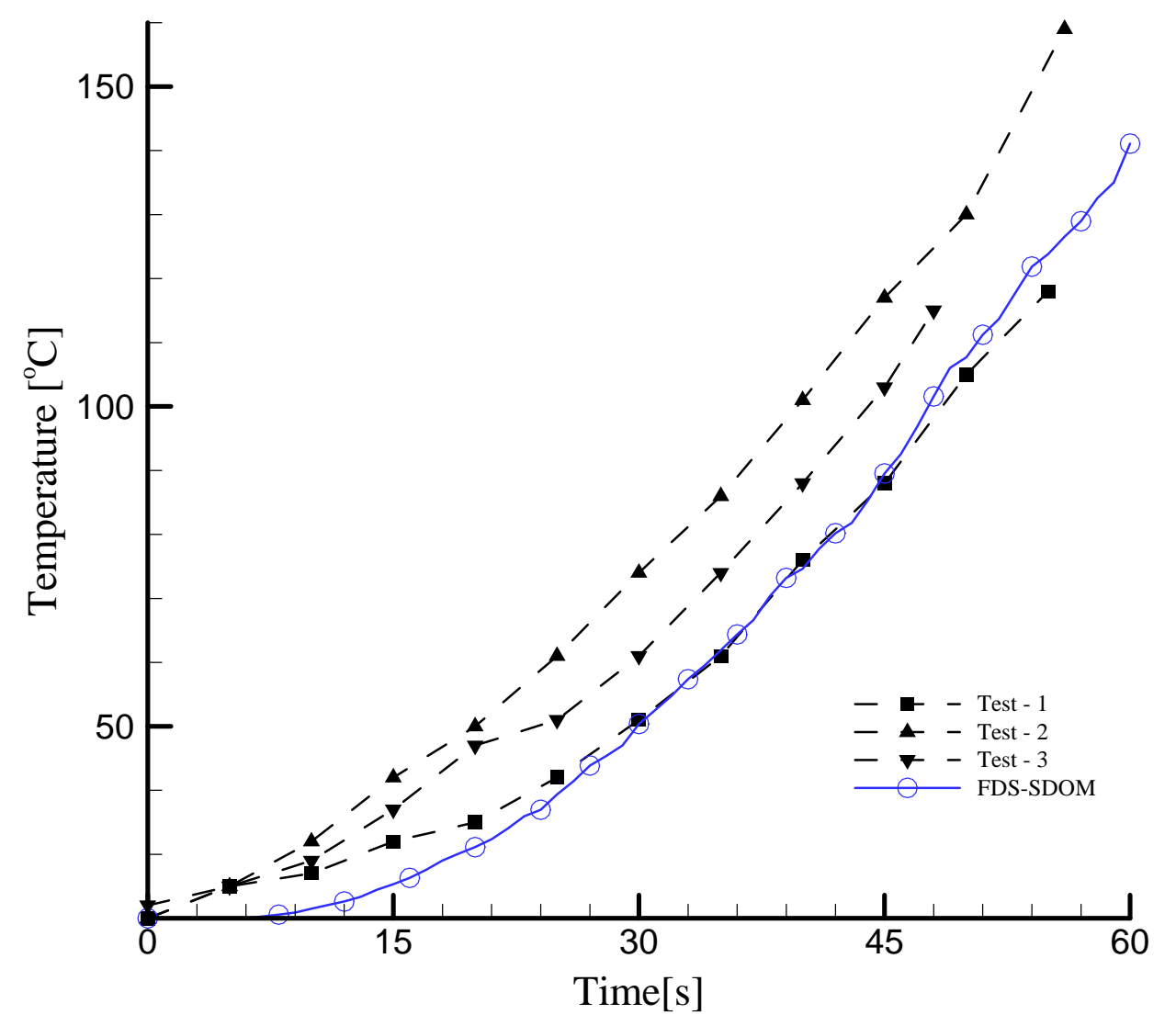

Fig. 7. Glass surface temperature predicted by FDS-SDOM and experimental data from [12]. Pool fire $30 \times 20 \mathrm{~cm}$.

\section{CONCLUSIONS}

A spectral radiative heat transfer model based on the discrete ordinates method (SDOM) previously developed by the authors has been implemented into the CFD code FDS 5.0 to predict the dynamic interation between glazing systems and fires. The paper has mainly reported the preliminary validation results of the new tool (referred to as FDS-SDOM) for some scenarios.

In the first validation study, FDS-SDOM is employed to predict the radiative heat fluxes in a wall with varying absorption coefficients typical of glass. The exact solution for this scenario has been reported in the literature. A comparative study between FDS-SDOM and the exact solutions shows that the code's predictions agree within $1 \%$ with the exact solutions. Simulations were also carried out for the same scenario using the original (unmodified) version of FDS (OFDS) which treats radiation in solids such as glass with a gray "two-flux" approach. OFDS solutions show large fluctuations with a maximum error of $13 \%$ in comparison to the exact solutions for the absorption coefficients investigated.

In the second validation study, FDS-SDOM is applied to the experimental scenarios of Harada et al. [15] and Skelly et al. [12]. In the first scenario where a glass pane is subjected to fire radiant heat [15], FDSSDOM predicts with a relatively good accuracy the glass surface transient temperature distribution. Application of OFDS is problematic for such a scenario where the glass spectral properties are to be accounted for. The second scenario considered is based on the compartment fire experiment of Skelly et al. [12]. A comparative study between FDS-SDOM and the experimental data [12] in terms of glass surface transient temperature show a relative good agreement between the two sets of data. 
The preliminary validation study presented shows the good potential of FDS-SDOM to predict the glass behavior in a fire environment. Work is in progress to apply the code to more fire/glazing scenarios for a better evaluation.

\section{ACKNOWLEDGMENTS}

The authors would like to acknowledge Pilkington Group Limited and the European Commission for financial support of this research.

\section{REFERENCES}

[1] Emmons, H.W., 1986. The Needed Fire Science. Fire Safety Science 1: 33-53. doi:10.3801/IAFSS.FSS.1-33

[2] Pagni, P.J., 1989. Fire Physics - Promises, Problems, and Progress. Fire Safety Science 2: 49-66. doi:10.3801/IAFSS.FSS.2-49

[3] Cuzzillo, B.R. and Pagni, P.J., (1998) Thermal Breakage of Double-Pane Glazing by Fire, J. Fire Protection Engineering 9: 1-11. doi:10.1177/104239159800900101

[4] Shields, T.J., Silcock, G.W.H., and Flood, M.F., (2001) Performance of a Single Glazing Assembly Exposed to Enclosure Corner Fires of Increasing Severity, Fire Mater. 25: 123-152. doi:10.1002/fam.764

[5] Pagni, P.J. and Joshi, A.A., "User’s guide to Break1, The Berkeley Algorithm for Glass Breaking in Compartment Fires”, National Institute of Standards and Technology Report NIST-GCR-91, Gaithersburg, MD, 1991.

[6] Dembele, S., Rosario, A.F., and Wen, J.X., (2007) Investigation of Glazing Behaviour in a Fire Environment using a Spectral Discrete Ordinates Method for Radiative Heat Transfer, Numerical Heat Transfer - Part B: Fundamentals 52: 551 - 568. doi: 10.1080/10407790701563672

[7] Dembele, S., Rosario, A.F., Wen, J.X., Varma, K.S., Dale, S., Wood, M., and Warren, P.D., 2007. Study of Glazing Behaviour in Fire Conditions Using Advanced Radiation Heat Transfer Model and Computational Fluids Dynamics. Proc. Seventh Asia-Oceania Symposium on Fire Science and Technology (in press).

[8] Gardon, R., (1956) The Emissivity of Transparent Material, J. American Ceramic Society 39: 278287. doi:10.1111/j.1151-2916.1956.tb15833.x

[9] McGrattan, K., Hostikka, S., Floyd, J., Baum, H.R., and Rehm, R.G. "Fire Dynamics Simulator, Version 5, Technical Reference Guide”, National Institute of Standards and Technology Special Publication 1018-5, Gaithersburg, MD, 2007.

[10] Kang, Y. and Wen, J.X., (2005) Large Eddy Simulation of a Small Pool Fire, Combustion Science and Technology 176: 2193-2223. doi:10.1080/00102200490515074

[11] Dembele, S., Wen, J.X., and Sacadura, J.F., (2000) Analysis of the Two-Flux Model for Predicting Water Spray Transmittance in Fire Protection Application, ASME Journal of Heat Transfer 122: 183-186. doi:10.1115/1.521454

[12] Skelly, M.J., Roby, R.J., and Beyler, C.L., (1991) An Experimental Investigation of Glass Breakage in Compartment Fires, J. Fire Protection Engineering 3: 25-34. $\underline{\text { doi:10.1177/104239159100300103 }}$

[13] Gardon, R., (1958) Calculation of Temperature Distributions in Glass Plates Undergoing Heat Treatment, Journal of the America Ceramic Society 41: 200-208. doi:10.1111/j.11512916.1958.tb13541.x

[14] Zel'dovich, Y.B. and Raizer, Y.P., Physics of shock waves and high-temperature hydrodynamic phenomena. (translated from Russian and then edited by W.D. Hayes and R.F. Probstein); Dover Publications, New York, 2002, 944 p. 
[15] Harada, K., Enomoto, A., Uede, K., and Wakamatsu, T., 2000. An Experimental Study On Glass Cracking And Fallout By Radiant Heat Exposure. Fire Safety Science 6: 1063-1074. doi:10.3801/IAFSS.FSS.6-1063 
